\title{
An Ethogram of Body Patterning Behavior in the Biomedically and Commercially Valuable Squid Loligo pealei off Cape Cod, Massachusetts
}

\author{
ROGER T. HANLON, MICHAEL R. MAXWELL, NADAV SHASHAR, ELLIS R. LOEW ${ }^{1}$, \\ AND KIM-LAURA BOYLE \\ Marine Resources Center, Marine Biological Laboratory, Woods Hole, Massachusetts 02543; and \\ ' Department of Biomedical Sciences, Cornell University, Ithaca, New York 14853
}

\begin{abstract}
Squids have a wide repertoire of body patterns; these patterns contain visual signals assembled from a highly diverse inventory of chromatic, postural, and locomotor components. The chromatic components reflect the activity of dermal chromatophore organs that, like the postural and locomotor muscles, are controlled directly from the central nervous system. Because a thorough knowledge of body patterns is fundamental to an understanding of squid behavior, we have compiled and described an ethogram (a catalog of body patterns and associated behaviors) for Loligo pealei. Observations of this species were made over a period of three years ( $\geq 440 \mathrm{~h}$ ) and under a variety of behavioral circumstances. The natural behavior of the squid was filmed on spawning grounds off Cape Cod (northwestern Atlantic), and behavioral trials in the laboratory were run in large tanks. The body pattern components - 34 chromatic (including 4 polarization components), 5 postural, and 12 locomotor-are each described in detail. Eleven of the most common body patterns are also described. Four of them are chronic, or long-lasting, patterns for crypsis; an example is Banded Bottom Sitting, which produces disruptive coloration against the substrate. The remaining seven patterns are acute; they are mostly used in intraspecific communication among spawning squids. Two of these acute patterns--Lateral Display and Mate Guarding Pattern-are used during agonistic bouts and mate guarding; they are visually bright and conspicuous, which may subject the squids to predation; but we hypothesize that schooling and diurnal activity may offset the disadvantage presented by
\end{abstract}

Received 1 February 1999; accepted 20 April 1999.

E-mail: rhanlon@mbl.edu increased visibility to predators. The rapid changeability and the diversity of body patterns used for crypsis and communication are discussed in the context of the behavioral ecology of this species.

\section{Introduction}

Cephalopods have a highly developed system of visual communication that is expressed mainly through the skin. The distinguishing features of this remarkable chromatophore system are its speed of change and the diversity of body patterns that each individual uses for either crypsis or communication (Hanlon and Messenger, 1996). A body pattern is defined as the total appearance of the animal at any given time, and includes the expression of the full complement of chromatic (i.e., color or visual), textural, postural, and locomotor components (see Packard and Hochberg, 1977; Hanlon and Messenger, 1996). Among the components of the body pattern, the most conspicuous are chromatic, although squids probably perceive intraspecific signals monochromatically because cephalopods are thought to be color blind (Hanlon and Messenger, 1996). These chromatic components are produced primarily by chromatophore organs and various reflective cells in the dermis, and they are discrete neural entities (just as postural, textural, and locomotor components are) because the chromatophore organs are controlled by radial muscles under the direct control of the posterior chromatophore lobes in the brain (e.g., Dubas et al., 1986). Most of the reflective cells are also controlled by the squid (Cooper et al., 1990). This neural control enables the cephalopod to change its appear- 
ance in a fraction of a second, depending upon the visual sensory input it receives during behavioral interactions. Few, if any, animals can match the speed of change and diversity of cephalopod signals, and the body patterns are used in most behavioral interactions, whether they be for competition for resources or mates, or interactions between predators and prey. We are documenting these diverse body patterns, focusing primarily on adult squids during their inshore migration every year.

Squids, like other cephalopods, are sensitive to the partial polarization characteristics of light (Saidel et al., 1983; Hanlon and Messenger, 1996; for a description of polarized light see Kattawar, 1994; Wolff and Andreou, 1995). Shashar and Hanlon (1997) described a few specific polarization components of squid and correlated these patterns with the distribution of iridophore cells in the animals' skin. In cuttlefish, partial polarization patterns have been associated with communication (Shashar et al., 1996). Since squids may use polarization patterns for intraspecific communication, and since polarization-sensitive predators may be looking for polarization contrasts to locate squid prey, we also document here some polarization components presented by the squid.

The long-finned squid Loligo pealei Lesueur, 1821, is a renowned model in neuroscience research. The third-order giant axon, its attendant giant synapse, the complex eye, and several other organ systems in $L$. pealei have been studied in detail for over 50 years at the Marine Biological Laboratory (MBL) in Woods Hole (see Gilbert et al., 1990). Although a great deal is known about the peripheral nervous system of $L$. pealei, little is known about the behaviors of this squid, which like most cephalopods, has an enormous brain relative to its body size. Loligo pealei is also a valuable commercial resource in the northeastern United States--worth about $\$ 30$ million annually (McKiernan and Pierce, 1995; NEFSC, 1995). Curiously, little is known about the ecology, life history, and behavior of this species (e.g., Verrill, 1880; Drew, 1911; Stevenson, 1934; Griswold and Prezioso, 1981; Summers, 1983; Gilbert et al., 1990; Brodziak and Macy, 1996). The present report is part of a broad-based study that focuses on sexual selection processes in $L$. peale $i$ from two perspectives: as a test of sexual selection theory (e.g., Hanlon, 1996; Hanlon et al., 1997) and as a study of the role that reproductive behavior plays in the life history and population dynamics of the species (Hanlon, 1998).

\section{Materials and Methods}

The behavior of Loligo pealei can be observed both in a natural setting and in the laboratory because the squids habituate quickly to divers and to laboratory surroundings.
Overall, $27.5 \mathrm{~h}$ of videotape were analyzed for body patterning and behavior.

During the months of May 1996, May 1997, and May 1998, 103 scuba dives were made on squid spawning grounds by RTH and NS off the southern arm of Cape Cod, Massachusetts. Depths ranged from 3-10 m and most sites were within $2 \mathrm{~km}$ of shore between Hyannis and Chatham. Spawning squids were found mostly in or near commercial weir traps whose inner pocket dimensions (or capture arena) were roughly $20 \mathrm{~m}^{2}$; often there were many thousands of squids in these traps, with a proportion of them actively engaged in reproductive behavior. Water temperatures ranged from about $4^{\circ}$ to $13^{\circ} \mathrm{C}$, currents were often strong, and visibility was usually poor. On about one-third of the dives, conditions were suitable for video. In total, $16.5 \mathrm{~h}$ of dive video were recorded, using video cameras (either analog or digital) in underwater housings, and analyzed, with multi-motion playback machines and high-resolution monitors.

Laboratory trials of mating behavior were performed from May through October in 1996, 1997, and 1998 in the Marine Resources Center of the MBL. Three large tanks were used, each measuring $3 \mathrm{~m}$ (diameter) by $1 \mathrm{~m}$ (height) and containing about $28,000 \mathrm{l}$ of seawater. Each tank had a substrate of mixed gravel and sand, and a continuous supply of ambient seawater. Animals were acquired by squid jigging (both at night and during the day) off the MBL research vessel Gemma in Vineyard and Nantucket Sounds. This method minimizes skin damage for maximal survival in captivity (see Hanlon et al., 1983). Squids were fed live fish (Fundulus sp.) daily. Trials involved from three to eight squids in various combinations of males and females. One set of trials was performed in an outdoor pond, $20 \mathrm{~m} \times 20 \mathrm{~m}$ $\times 1 \mathrm{~m}$ deep, at the Environmental Systems Laboratory of the Woods Hole Oceanographic Institution. The squids were observed for $440 \mathrm{~h}$ in captivity, 11 of which were recorded on video.

All videos were reviewed multiple times, each time looking for only one category of component (i.e., first viewing for chromatic components, second viewing for postural components, third viewing for locomotor components). In the laboratory, chromatic, postural, and locomotor components were recorded on separate data sheets each time they were seen. A chromatic component was recorded if it was expressed for at least $2 \mathrm{~s}$; locomotor and postural components were recorded if they were performed for at least $3 \mathrm{~s}$. All chromatic components were illustrated using a computer graphics program.

Polarization components were recorded using a video polarimeter based on a standard three-tube ENG camera (JVC BY-110) that uses a dichroic prism block for color separation. The dichroic prism has been replaced with a custom-made neutral prismatic splitter (Richter Enterprises, 
Manhattan Beach, CA) such that each of the three video channels receives $1 / 3$ of the broad-spectrum image input. Since this assembly lacks the color-trimming filters cemented to the original dichroic prism, magnification errors due to pathlength differences were corrected with small quartz discs of appropriate thickness. A small disc of sheet polarizer (Polaroid, HNP'B) was placed immediately in front of each camera tube to impart polarization sensitivity to the channels. The orientation of the polarizers was adjusted so that the color channels now encoded $0^{\circ}, 45^{\circ}$, and $90^{\circ}$ polarization images. The camera electronics encode the three polarization channels as if they were color, making it possible to store all the data on a regular portable videocassette recorder and allowing for immediate viewing of a pseudocolor polarization image on a color monitor. Nonpolarizing elements of the scene have no color, whereas polarizing elements do. The signal in all three channels is identical, and the output of the tubes was adjusted to give white for a saturating faceplate intensity. A polarizer placed in front of the lens such that horizontally polarized light is freely transmitted produces the following normalized signals in the three "color" channels: the R channel signal is 1 , the $\mathrm{G}$ channel is 0.707 , and the $\mathrm{B}$ channel is 0 . Monochromatic images of the same scene, taken from the three channels separately, were transferred through a frame grabber into the computer and their linear polarization characteristics were analyzed following procedures in Cronin $e t$ al. (1994). This camera is better suited than previously described polarimeters (Cronin et al., 1994; Wolff and Andreou, 1995; Horvath and Varju, 1997) for recording the polarization patterns of moving animals, because it provides true instantaneous measurements. Technological limitations made it impossible to get the camera in an underwater housing; thus measurements were limited to the laboratory. Furthermore, the light conditions during measurements had to be precisely controlled, thereby allowing only $3 \mathrm{~h}$ of recorded footage. During these periods, the squids exhibited only a few behaviors that included fighting, mate guarding, and egg laying.

\section{Ethogram}

We constructed an ethogram for Loligo pealei on the basis of our field and laboratory observations. The components and body patterns identified (Table I) represent a segment of all behaviors, especially those related to reproduction. In fact, because of the size of the sample, most of the patterning components of the species were probably identified. The more than $440 \mathrm{~h}$ of observation far exceed the observation periods in other published accounts of $\mathrm{LO}^{-}$ ligo spp. (e.g., Hanlon, 1982, 1988; Hanlon et al., 1983, 1994; Porteiro et al., 1990).
The chromatic components of the ethogram are illustrated in Figures 1 and 3, and some of the postural components are shown in Figure 2. Unlike octopuses and cuttlefishes, loliginid squids do not show textural components in the skin. Table I, which lists all components, includes the number of times that we counted a component on videotape or from observation notes, giving an impression of how commonly it occurs. Unless otherwise indicated, all components and body patterns were shown by both sexes.

\section{Light chromatic components}

Chromatic components are produced mainly by the action of dermal chromatophore organs, which number in the hundreds of thousands in an adult squid. Loligo pealei has three color classes of chromatophores: yellow, red, and brown. Expansion of the chromatophores darks the skin, while retraction of the chromatophores (and the resultant expression of underlying iridophores) produces a lightening or even brightening effect. Intense darkness produced by maximal expansion and intense brightness produced by maximal retraction mark two ends of a chromatic continuum, and thus it is somewhat arbitrary to assign a component to light or dark. Some of these components are common to other Loligo spp., as described by Hanlon (1982) for Loligo plei, by Porteiro et al. (1990) for Loligo forbesi, and by Hanlon et al. (1994) for Loligo vulgaris reynaudii.

Clear is retraction of all or most chromatophores, thus rendering the animal translucent in clear water or white in murky water. In clear water, when viewed against a sand bottom or laterally against the aquatic background (Fig. 2B), the translucence renders the squid cryptic, or camouflaged, and often the Dorsal iridophore splotches are expressed simultaneously. Internal organs, such as the red accessory nidamental gland in females, are often visible. In murky water, Clear appears bright white in most lighting circumstances (i.e., the brightness surpasses the albedo of the greenish water, producing a whitish color). In the immediate vicinity of egg beds, the white form of Clear seems to function as an intraspecific signal to repel other squids; a squid displaying this component is almost always engaged in mate guarding, egg laying, or agonistic bouts (see Fig. 2C). White arms/head results from variable retraction of chromatophores on the head and arms (three variations are illustrated in Fig. 1). This component sometimes preceded all white (or clear) in intraspecific encounters; thus, it appears to be a milder signal of alarm or repellent to approaching squids (Fig. 2G). White head/arms is most common in paired females near eggs and is seen when unpaired males approach. White dorsal stripe is retraction of chromatophores along a dorsal mantle that is otherwise dark; the stripe may be short or long (Fig. 1). It has been seen in 
Table I

Body patterns and their components in the squid Loligo pealei; compare Figure I

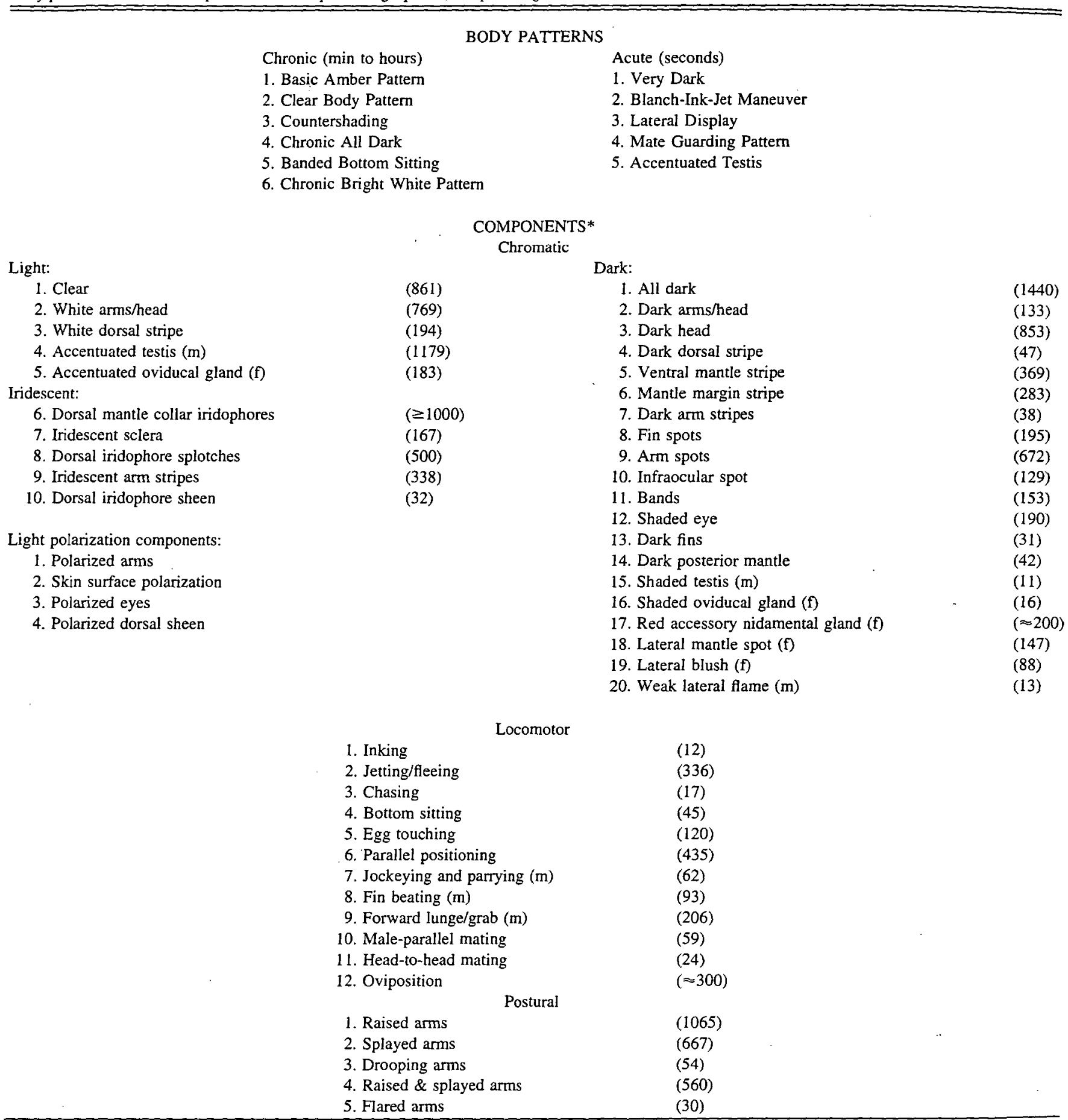

* Letters in parentheses indicate that the component is sex-specific: $f=$ female; $m=$ male. Numbers indicate how many times each component was observed on video or in laboratory trials. 

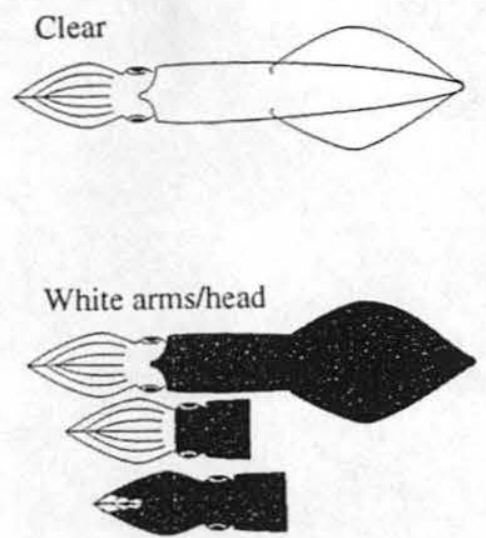

White dorsal stripe

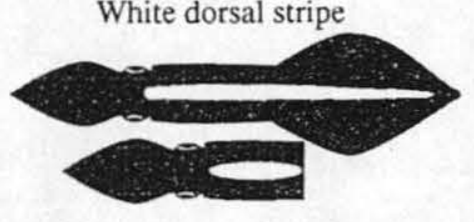

Accentuated testis (m)

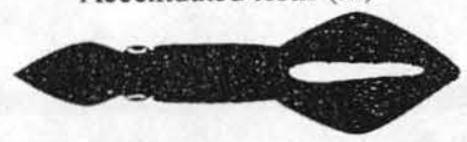

Accentuated oviducal gland (f)

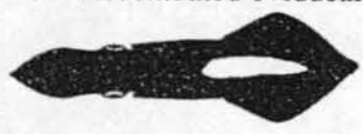

Dorsal mantle Iridescent sclera collar iridophores

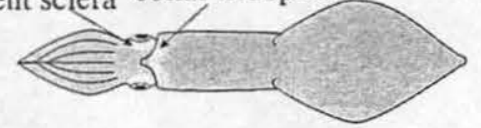

Dorsal iridophore splotches

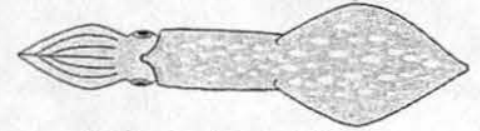

Iridescent arm stripes

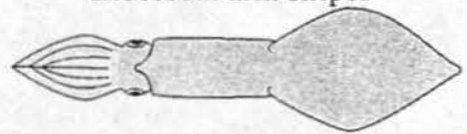

Dorsal iridophore sheen

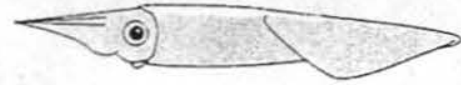

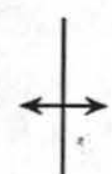
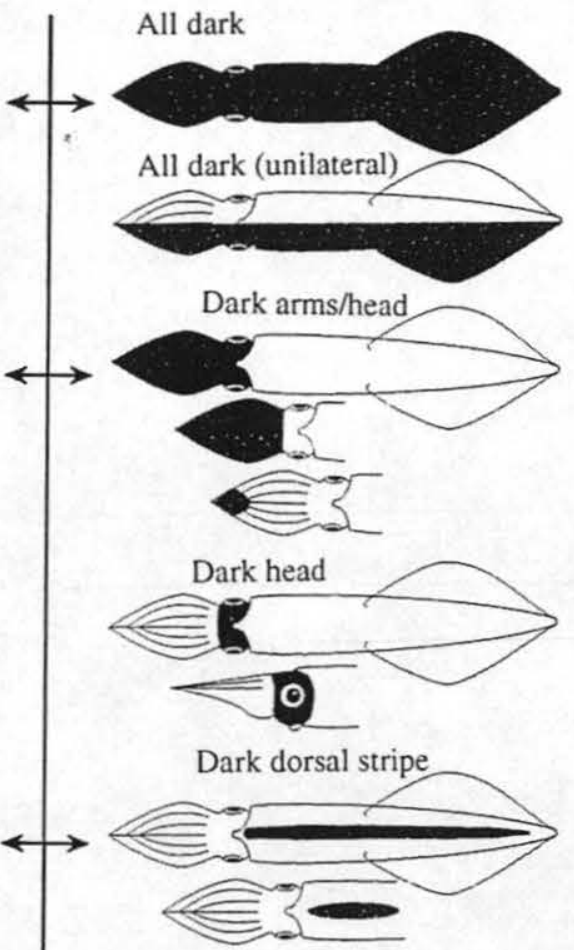

Shaded testis (m)

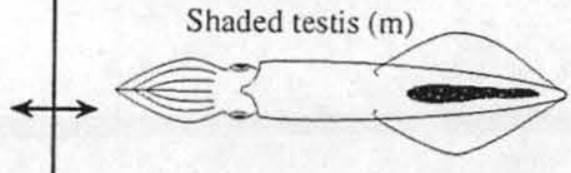

Shaded oviducal gland (f)

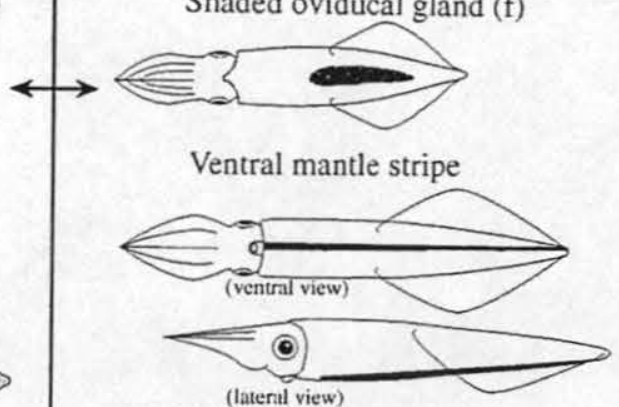

Mantle margin stripe
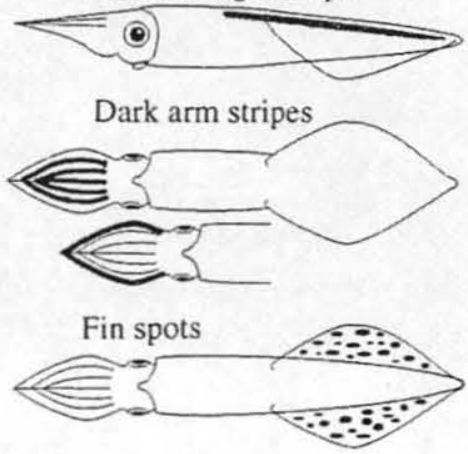

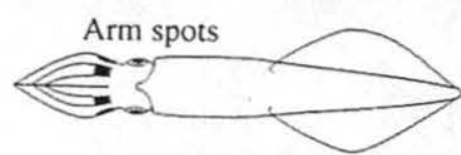

Infraocular spot

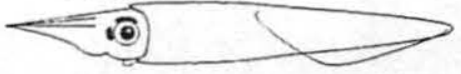

Bands (with variations)
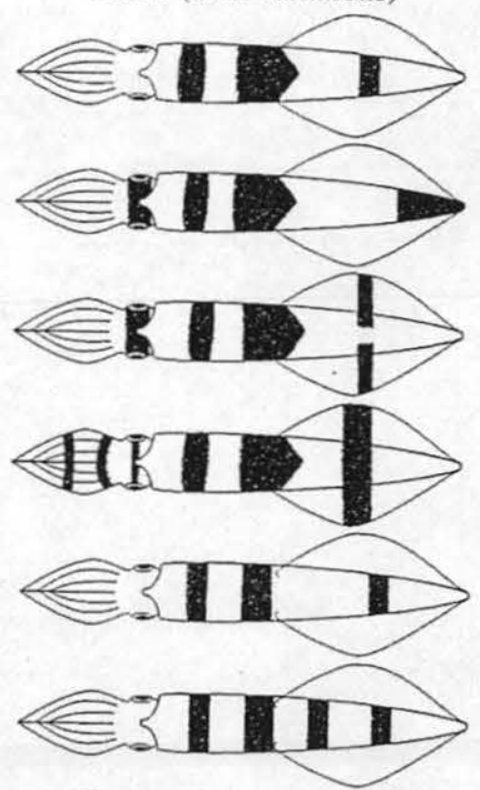

Shaded eye

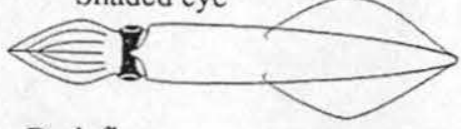

Dark fins

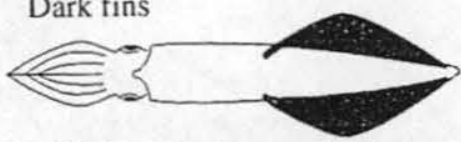

Dark posterior mantle

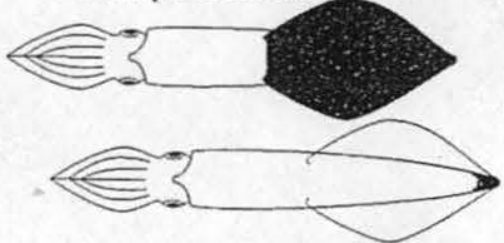

Red accessory nidamental gland (f)

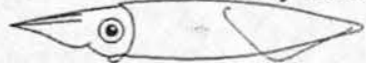

Lateral mantle spot (f)

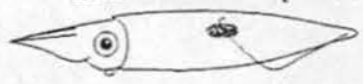

Lateral blush (f)

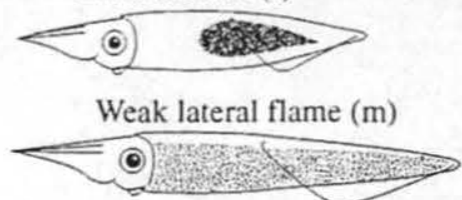

Figure 1. Chromatic components of body patterning in the squid Loligo pealei. The arrangement generally follows Table I and the text. 

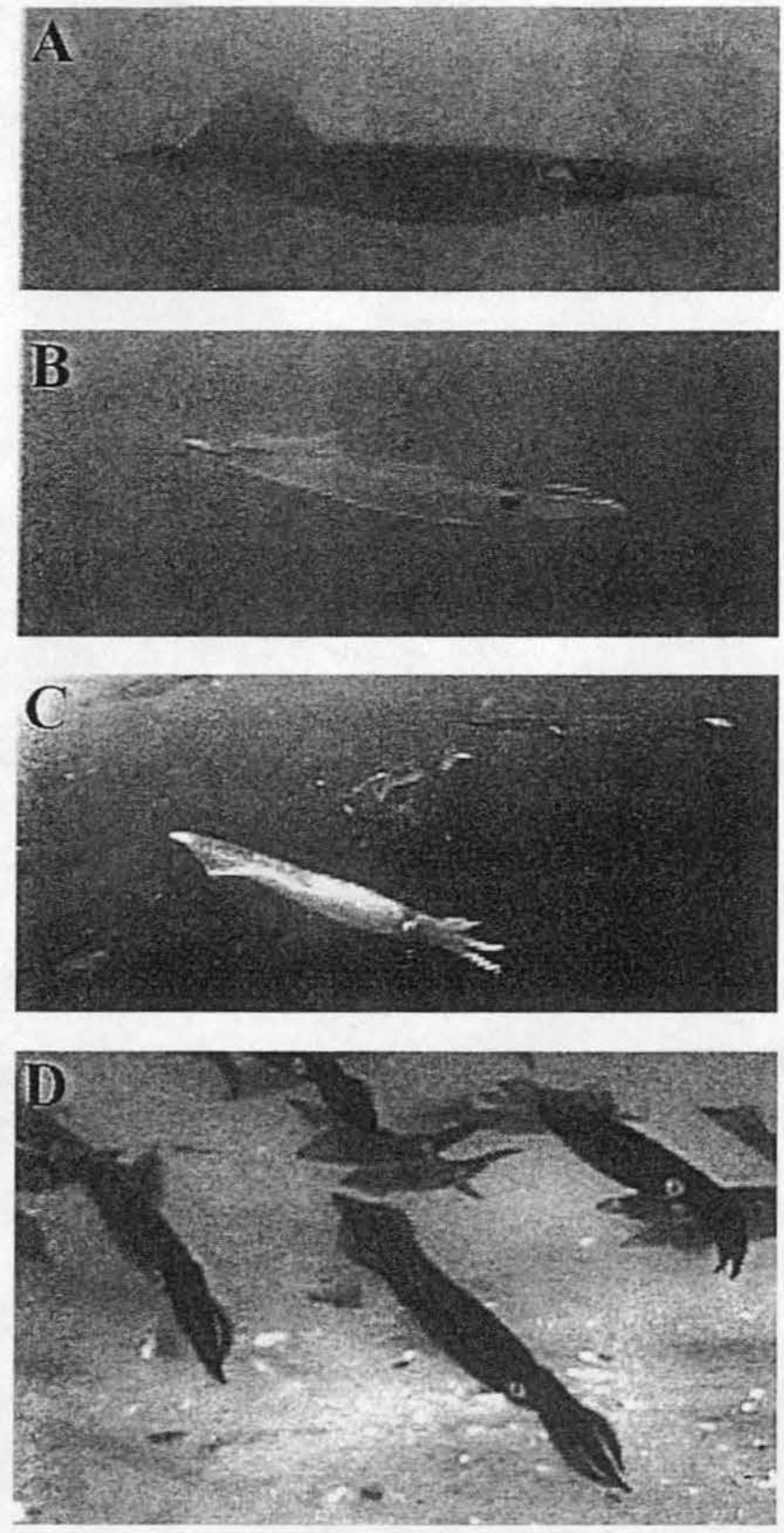
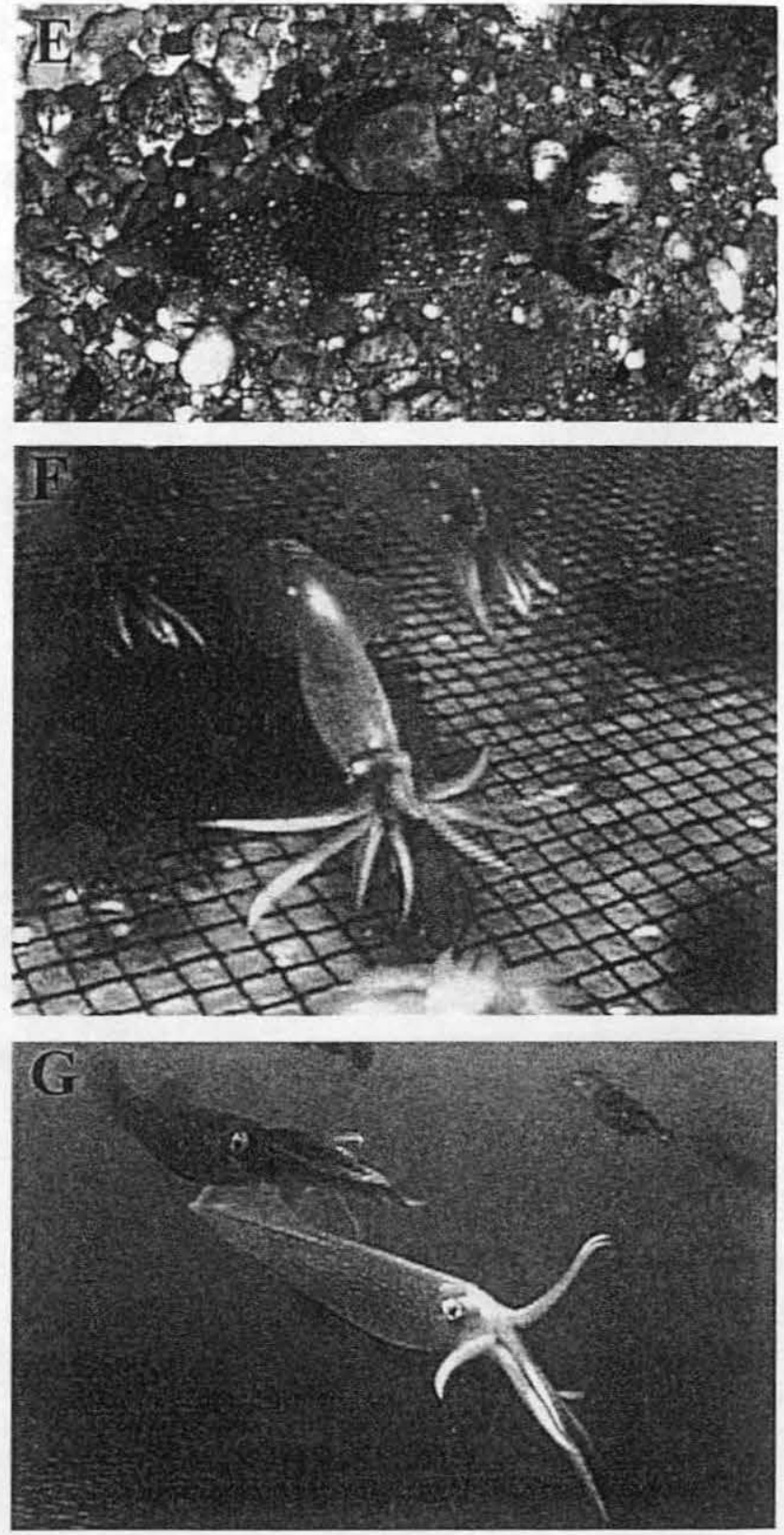

Figure 2. Underwater video images of selected components and body patterns of Loligo pealei. (A) The chronic Basic Amber Pattern. (B) The chronic Clear Body Pattern. (C) The chronic Bright White Pattern amidst other squids in Basic Amber. (D) The chronic All Dark pattern viewed against a sand substrate. (E) The Banded Bottom Sitting pattern showing disruptive coloration against a gravel substrate. (F) Acute Mate Guarding Pattern shown by a large consort male (female is just below him) showing the Splayed arm posture and the Accentuated testis chromatic component. (G) Raised arms postural component in a male that also shows the chromatic component of White arms/head; he is directing this signal to the lone male at upper left as he guards his female mate (barely visible behind him). 


\section{Intensity}

A
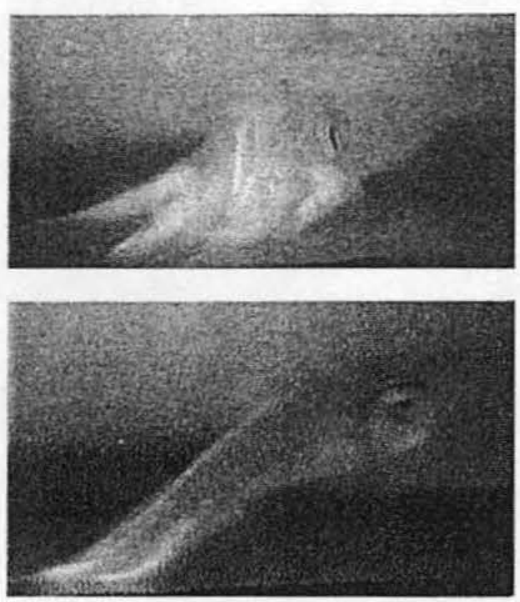

C
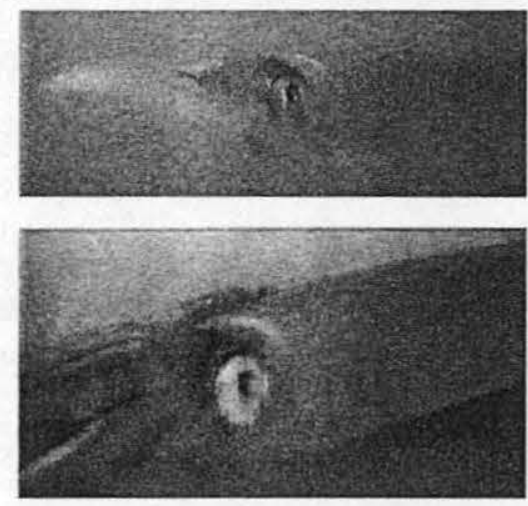

E

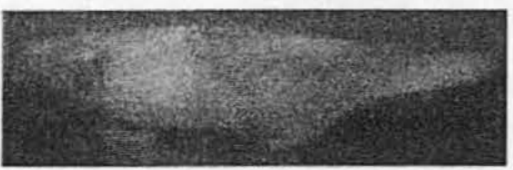

Partial polarization
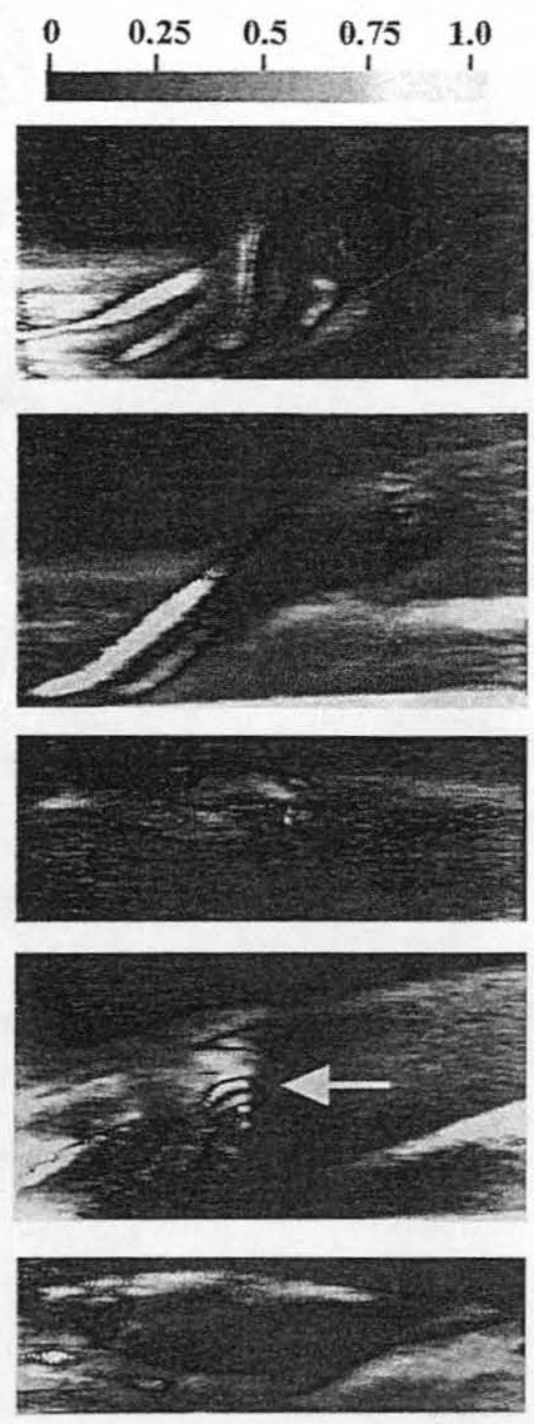

Orientation of polarization
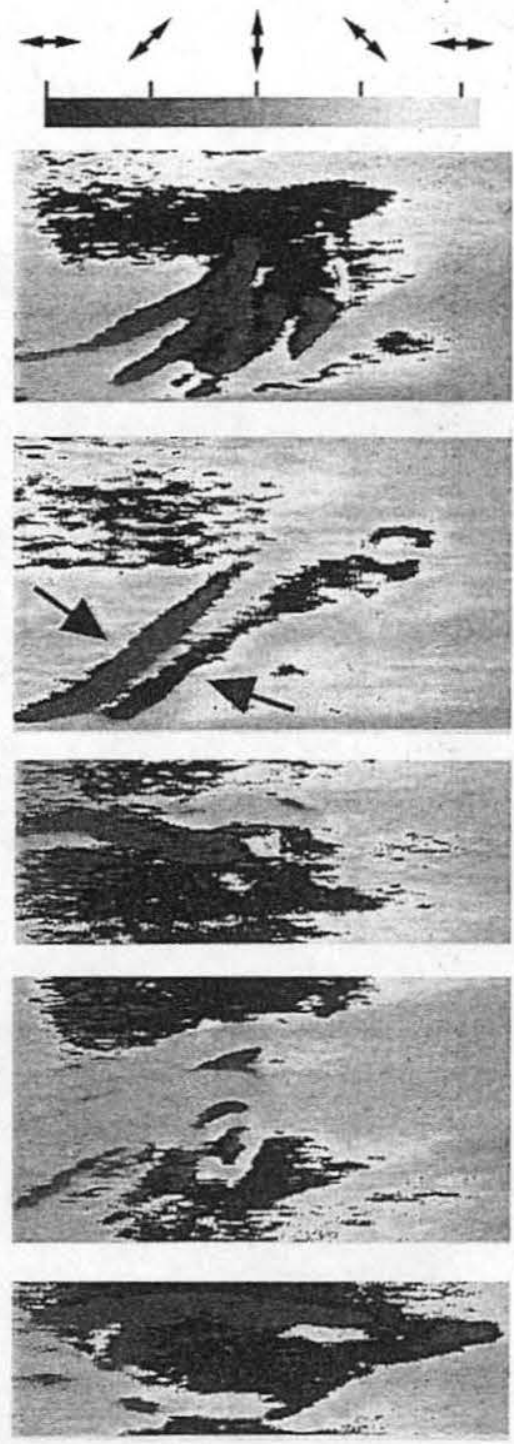

Figure 3. Selected images demonstrating the main sources of polarization components in adult squids. LEFT: Black-and-white images of the squid. CENTER: Partial polarization images in which black represents unpolarized light -0 , and white represents full linear polarization -1 . RIGHT: Orientation of polarization; horizontal polarization is coded into white or black, and vertical polarization into $50 \%$ grey. Special iridophores on the arms create the predominant components (A, B), where the partial polarization can exceed 0.75 . The orientation of polarization can be equal on all arms (A) or it can vary between them (B, indicated by arrows). Structural reflection from the skin-water interface can produce a polarization pattern that changes with the animal's motion (C). The reflection from the sclera of the eye may be highly polarized (D, arrow). The top of the mantle of the squid occasionally reflects light that is partially polarized (E). This polarization may arise from structural reflection, as in C, or from reflection by the iridophores on the squid's mantle or splotches (Shashar and Hanlon, 1997). 
consort males when an intruder male approaches. Accentuated testis is a male-only component shown when the chromatophores directly above the testis are retracted while the squid mantle is otherwise dark, thus accentuating the whiteness of the organ (Fig. 2F). This component was seen frequently in single or mate-paired males when reproductive behavior was actively occurring in the school. Accentuated oviducal gland is a female-only component analogous in form and function to Accentuated testis in the male. This was often seen in females paired with consort males. All of these light components except White dorsal stripe have been seen commonly in other Loligo spp.

\section{Light iridescent chromatic components}

Each of the light iridescent chromatic components is common to Loligo spp., and comparable color images may be viewed in Hanlon (1982). Dorsal mantle collar iridophores are on the anteriormost portion of the mantle, and they appear as bright yellow or pink iridescence; this component tends to produce disruptive coloration by breaking up the longitudinal aspect of the squid's body. It and the next component are usually seen on calm squids near the bottom in the Clear pattern. Dorsal iridophore splotches occur on the dorsal mantle and head. They are a distinctive yellow or golden color, and they help to produce general camouflage (Fig. 2E). Iridescent arm stripes extend most of the length of the first three pairs of arms. These are usually expressed lightly during camouflage in the Clear pattern, but during agonistic encounters they can be expressed very brightly (see color illustration in Hanlon, 1982). Iridescent sclera is the bright silver iridescence on the back (or sclera) of the eye; squids have the ability to obscure this with chromatophores with the Shaded eye component. Dorsal iridophore sheen is somewhat rare and is only noticeable from the side. Its function is unclear but may aid camouflage in open water by disrupting the body shape. None of these are unique to $L$. pealei but are shared by other Loligo spp.

\section{Light polarization chromatic components}

These linear polarization components are newly described for Loligo spp. Polarized arms are highly polarized reflections that create the most conspicuous component of polarization (Fig. 3A, B). This component often exceeds partial polarization of 0.75 , which is noteworthy because Flamarique and Hawryshyn (1997) showed that the natural underwater light field rarely exhibits partial polarization as high as 0.67 . The orientation of polarization can be equal in all arms (Fig. 3A), or it may differ between arms (Fig. 3B).
Skin surface polarization results from the difference in refractive indexes between the squid's body and the water, so that light reflected from any area of the skin may be partially polarized (Fig. 3C). However, the partial polarization in this case is mostly low, rarely reaching 0.5 . Polarized eyes result from reflection by iridophore cells that surround the eye (Fig. 3D, arrow). The dorsal mantle occasionally reflects light that is partially polarized, resulting in Polarized dorsal sheen. The orientation of polarization can vary, reaching 20 degrees from horizontal. This polarization reflection corresponds to the area of the Dorsal iridophore sheen, although the two components do not always coincide in time. The source of this polarization component can be either reflection from iridophores on the mantle or Skin surface polarization. Owing to the limitations of the equipment used to record polarization patterns, these are probably not the only polarization components that squids can show.

\section{Dark chromatic components}

All dark is the opposite of Clear: all or most chromatophores are expanded to some degree. The maximal expression of All dark (Fig. 2D) produces an overall deep brown coloration; it is characteristic of alarmed squids. However, the chromatophores need not be maximally expanded, and thus there are ranges of darkness. Often squids are in a "normal" or "basic" coloration that is roughly between Clear and All dark, producing an overall amber body pattern (Fig. 2A). There is also a striking unilateral expression of All Dark (Fig. 1). Dark arms/head is variable in expression (see Fig. 1) and is opposite to White arms/head. It is seen typically in mating pairs and may represent a mild state of alarm. Dark head is expansion of all the chromatophores around the head of the animal (but not the arms), causing the head to appear almost black. This component is frequently seen in mate pairs near the egg mop and probably represents a low-grade alarm signal.

Four striped components occur in L. pealei, one used for crypsis and three used during intraspecific agonistic contests. Dark dorsal stripe extends halfway or fully down the mid-dorsal mantle. Seen mainly on calm squids, it apparently aids camouflage because it covers some of the bright organs such as the testis, oviducal glands, and ink sac. Ventral mantle stripe is a thin, distinct line of fully expanded chromatophores. $L$. pealei, in contrast to $L$. plei but in common with $L$. vulgaris reynaudii, $L$. vulgaris, and $L$. forbesi, shows no protrusile flap of skin when exhibiting this component (Hanlon, 1988; Hanlon et al., 1994). The function of this component is uncertain, but it is seen commonly on mating pairs and on males during mate guarding. Males often swim just above females, and pairs are frequently approached by other squids from below, so the ventral 
positioning of this visual signal may be useful. It is also possible that the stripe helps disrupt the body form when viewed from below by predators. Mantle margin stripe is a dark line running along the fin insertion. It was seen most often as a mild reaction to disturbance or alarm during agonistic bouts, and was usually expressed in conjunction with Ventral mantle stripe, Fin spots, and weak Lateral flame (see below). Dark arm stripes are variable, being expressed either along the third pair of arms or along pairs 1,2 , and 3. This uncommon component was seen on a female that also expressed Dark fins (also uncommon, see below) just before a male mated her, and as another mating pair bumped into them. Thus it seems to be an expression of alarm when all three arm pairs are darkened. The simultaneous expression of stripes on three arm pairs has not been reported for squids.

Three spotted components are expressed during alarm or threat situations, mainly intraspecifically, and can be shown unilaterally on the side towards the other squid. Fin spots are a collection of small circular and oval dark spots scattered across the fins. This component is seen mostly during agonistic bouts or rarely when an aggressive male comes close by. Arm spots are small and occur at the base of the third arms, the second arms, or both. This component is seen on males during mate guarding and at the early stages of agonistic encounters; it probably constitutes a low grade of alarm (see also Arnold, 1962, 1990). Infraocular spot appears directly in front of the eye and has variations, including a circular shape that looks like an eye ring. The avenue of achieving signals of "increasing alarm" appears to be Arm spots $>$ Infraocular spot $>$ expanded to eye ring $>$ Dark head.

Various other dark components include two for crypsis and four for intraspecific alarm situations. Bands are variable (see Figs. 1 and 2E) and may occur on the fins, head, or arms. First reported by Stevenson (1934), this component is seen typically in calm, bottom-sitting squids and functions as disruptive coloration to break up the longitudinal outline of the squid. Shaded eye is a transverse head bar of expanded chromatophores that may aid crypsis by covering the bright Iridescent sclera of the eyes. Dark fins occur when all fin chromatophores are expanded maximally; it is not common but has been seen on females that are alarmed. Dark posterior mantle is similar to Dark fins, but the mantle chromatophores are expanded; it may be the next stage of alarm after Dark fins.

Several dark components associated with reproductive behavior complement the light components Accentuated testis and Accentuated oviducal gland. Shaded testis and Shaded oviducal gland are selective expansion of chromatophores over the testis or oviducal gland. Both are often indistinct and serve to mask these bright white organs, thus aiding crypsis. However, the complementary "shading/ac- centuating" allows rapid signaling. The Red accessory nidamental gland can be seen through the translucent mantle and occurs only in fully mature females, so it may be a part of communication even though it is internal. Since it turns red only upon attainment of full sexual maturity, it may be a sign of female sexual maturity or even receptivity. Lateral mantle spot is a female-only component expressed as a small intense dark spot of chromatophores near the anterior fin insertion. It coincides roughly with the position of the Red accessory nidamental gland, and the two may function together in some way. The Lateral mantle spot is seen only when the female is paired with a large consort male, and could indicate either receptivity or rejection. Lateral blush is a female-only component expressed unilaterally as a diffuse dark area on the lateral mantle. It may be comparable to a variety of similar components shown by female squids, and it may function as a repellent to courting males (Hanlon and Messenger, 1996: their fig. 6.21).

Weak lateral flame is a male-only component produced by longitudinally oriented rows of partly expanded chromatophores. It is seen during low-grade agonistic contests. There are several variations of this component in other Loligo spp., the most well developed and dramatic of which is in Loligo plei (Hanlon, 1982; DiMarco and Hanlon, 1997). In Loligo vulgaris, Loligo vulgaris reynaudii, and Loligo forbesi there are Lateral mantle streaks that are arranged a bit differently in the skin, but they all function to provide a lateral signal to an opposing male. Loligo pealei has perhaps the weakest expression of this component, while $L$. plei has the strongest.

\section{Postural components}

Five postural components are expressed through the arm positioning of Loligo pealei. They are generally comparable to postures seen in other Loligo spp. Raised arms (Fig. 2G) is the unilateral or bilateral raising of the first pair of arms, which may be light or dark, and is seen in both males and females on the mating grounds. This component appears to be a signal of alarm during agonistic contests. It was previously reported by Arnold $(1962,1990)$. Splayed arms (Fig. 2F) is a posture in which all eight arms are spread and flattened on the horizontal plane. This posture is expressed by both sexes but is most common in males that use it to guard female mates they are escorting to egg mops. Raised and splayed arms are a combination of the previous postures in which the arms are all splayed except for the first pair, which is raised; it is a strong signal of alarm used when a rival male approaches closely. Drooping arms in a swimming squid is a posture in which all the arms appear relaxed and hang downward, but its function is unknown. Flared arms is a rare posture in which all of the arms are held 
stiffly outward in a radial manner; it is seen during highly aggressive agonistic encounters between two males, and during mate guarding.

\section{Locomotor components}

Inking is the expulsion of ink mixed with mucus, either in small puffs or as a large dense cloud (Hanlon et al., 1994). Inking is often followed by Jetting/fleeing, which is a rapid jet-propulsed escape used in avoidance of both predators and conspecifics. Females often jet from males that try to swim with them or copulate with them. Chasing occurs when one squid actively pursues another, usually in forward swimming. In most cases a male is pursuing another male at the conclusion of an agonistic bout. Bottom sitting occurs when a squid rests on the substrate (Fig. 2E). Egg touching consists of contacts with an egg mop by both males and females. Contact ranges from brief, exploratory touches to embraces of an egg capsule with all of the arms. Females usually lay eggs on existing egg mops, and touching may be a way of assessing the egg-laying substrate. Males commonly touch eggs, and touching is often followed by highly aggressive agonistic bouts (Hanlon, 1996), suggesting that the eggs provide a visual, tactile, or perhaps chemosensory stimulus. Parallel positioning occurs when two animals are hovering or swimming parallel to one another in the same direction, within one body length or each other. Courting pairs maintain this position, and agonistic encounters begin with this movement. Jockeying and parrying ( $\mathrm{m}$, males only) occur when two males maneuver to get next to a female. A successful paired male will often ward off (or parry) the jockeying movements of the unpaired male in a long sequence of swimming maneuvers. Fin beating $(\mathrm{m})$ occurs in the parallel position when two males maneuver themselves so that they are beating their fins against each other. This is a physical and escalated stage of an agonistic context, but it results in no obvious physical damage. Forward lunge/grab $(\mathrm{m})$ is a short, fast movement to bluff or grab another male during agonistic contests. The grab sometimes results in grappling in which the squids attempt to bite each other. It is rare and is the highest escalation of a fight. Male-parallel mating occurs when the male positions himself under the female and grasps her anterior mantle to pass spermatophores into her mantle cavity. Head-to-head mating occurs when a male and female face each other, and the male grasps the female's arms. Spermatophores are placed in a seminal receptacle below the mouth (Drew, 1911). Oviposition (f, females only) occurs when the female extrudes a single egg capsule and affixes it to the substrate or to existing communal egg masses; she does not hold the egg capsule for long. Among these, egg touching is newly described for Loligo spp., although other species do this to varying degrees.

\section{Body patterns}

Chronic patterns last for minutes or hours. There are four general chronic body patterns that are common on calm squids and function as crypsis. The Basic Amber Pattern (Fig. 2A) is the most common and long-lasting body pattern observed in Loligo pealei, and it occurs while the squids are hovering, gently rocking back and forth, or swimming slowly. It is characterized by a partial expansion of all chromatophores (i.e., the All dark component). Apparently the squid detects the albedo in the immediate vicinity and neurally adjusts chromatophore expansion to match it, thus achieving crypsis by matching its surroundings. This pattern can grade into a lighter Clear Body Pattern (Fig. 2B) with expression of the Dorsal iridophore splotches; this is seen both when squids are swimming just above the substrate and when they are in the water column. Another subtle variation of these two patterns is Countershading, in which the chromatophores on the dorsal surfaces of the squid are in a rather uniform light expansion (as in Clear or Basic Amber) while the ventral portions of the animal are light (probably with help from the many iridophores in the dermis; see Cooper et al., 1990; Hanlon et al., 1990) to eliminate the shadow. In the natural environment, squids only a few meters away blend in almost perfectly with the water column. A more unusual situation occurs when a whole school of squids go into the Chronic All Dark Pattern (Fig. 2D), which is not cryptic at all. The function of this pattern is unknown, but we have observed it several times in the natural habitat on many hundreds of calm squids hovering in large schools in the water column. The Banded Bottom Sitting pattern (Fig. 2E) is very common and consists of Bands with Dorsal iridophore splotches and Bottom sitting. The pattern provides excellent crypsis through disruptive coloration because the bands break up the longitudinal shape of the squid, and the pattern has variations in the banding. To our knowledge, $L$. pealei is the only loliginid squid that commonly sits on the substrate, al though Loligo forbesi was observed to bottom sit in the laboratory on rare occasions (Porteiro et al., 1990).

Around egg beds, many squids that are actively engaging in sexual selection behavior remain in the Chronic Bright White Pattern (Fig. 2C), which is visually conspicuous. This pattern has many variations, but the most common one is seen on mating pairs near the egg beds. Males that are mate guarding are in Clear, Raised arms, White arms/head, Arm spots, and sometimes Dark head. Females that are being guarded are in Clear with Dark 
head, and sometimes Raised arms. In both cases the testis and the oviducal glands are clearly visible through the mantle. It is noteworthy that unpaired males (whether large or small) moving amidst a school of reproductively active squids are not in this bright white pattern, yet if a large male wins an agonistic contest and pairs with the female he will immediately go into the Chronic bright white pattern.

Acute patterns last for seconds or rarely for minutes and are seen during intra- and interspecific interactions. Very Dark has two variations. The first is a brief flash to a conspecific or an interspecific threat (e.g., to a person in the laboratory or a fish in the field). The second variation shows several flashes over a 5-s period, producing a strong Deimatic effect that startles or bluffs (see Hanlon and Messenger, 1996). The Blanch-Ink-Jet Maneuver may be universal among squids: the animal blanches Clear and jets away (usually backwards, but sometimes forward) while ejecting ink in a pseudomorph that remains in the approximate position from which the squid started the maneuver. This is a typical secondary defense against predation or threat (when, for instance, the primary defense of crypsis fails). Such behavior is called Protean behavior because the variable and erratic escape response upsets target prediction by the attacker (Driver and Humphries, 1988).

Lateral Display is a complex set of behaviors performed only by males during agonistic contests. There is some stereotypy, although it is by no means a fixed sequence (see Hanlon and Messenger, 1996; DiMarco and Hanlon, 1997). It begins with Parallel positioning by two males and then includes various visual signals including Arm spots, Infraocular spot, Fin spots, Mid ventral stripe, Weak lateral flame, and Raised and splayed arms. The overall base coloration of the body is bright white; this, because it is "turned on" so quickly as the chromatophores retract when a contest begins, gives the optical illusion of flashing. The ensuing dynamic interactions between the males include flashing and escalation to Fin beating followed by Jockeying on the part of the intruder male to get near the female, and Parrying by the paired male to fend off the intruder. Mate Guarding Pattern (Fig. 2F) is shown by paired consort males that are approached either by paired males or by single large or small males that may be seeking an extrapair copulation. The male hovers directly between his mate and the approaching male, maintaining a bright white coloration with Arm spots and maximally Splayed arms; the Accentuated testis is often conspicuous, especially if the male goes dark or amber briefly (Fig. 2F). Accentuated Testis is a single component that can and does act as a body pattern, and it is particularly common on small sneaker males that swim around spawning areas attempting extrapair copulations. This pattern is often shown with the All dark component, but it may also be paired with Basic Amber. Although we list it as an acute pattern, it can sometimes be shown often enough to be considered chronic.

\section{Comparisons With Other Loliginids}

\section{Sympatric Loligo pealei and Loligo plei}

The components of body patterns were compared between these species by Hanlon (1988) before detailed information on Loligo pealei was available. The importance of these comparisons is that the species are nearly indistinguishable morphologically at hatching (McConathy et al., 1980), as juveniles (e.g., Cohen, 1976), or even at adult size (Vecchione et al., 1998), and fisheries statistics usually lump the two species together in landing records. Hanlon (1988) should be consulted for many comparisons of these two species; only corrections or additions to that paper are discussed here. First, Accentuated testis and Accentuated oviducal gland (and their respective shaded counterparts) occur in both species, so these components cannot be used to distinguish them. Second, Lateral blush has now been seen in both species, but the Lateral mantle spot of female $L$. pealei seems to be unique. Third, Dark arm stripes in $L$. pealei seem distinctive. Fourth, Fin spots in $L$. pealei are strikingly distinguishable from Stitchwork fins in L. plei. Fifth, the bands are more variable and perhaps distinctive in L. pealei. The Lateral Displays of the two species are clearly different, especially the Mid ventral ridge and the dramatic Lateral flame of $L$. plei compared to the Mid ventral stripe (i.e., no ridge of extended skin) and the weak Lateral flame of L. pealei. Conversely, the bright white Mate Guarding Pattern of $L$. pealei seems distinctive, although field observations of natural spawning in $L$. plei would be needed to confirm this difference.

\section{Loligo forbesi and Loligo vulgaris reynaudii}

In general, Loligo pealei is comparable in the content and diversity of its patterning with other Loligo spp. Porteiro et al. (1990) provided an ethogram of $L$. forbesi based on limited laboratory observations in the Azores Islands, and Hanlon et al. (1994) provided an ethogram of L. vulgaris reynaudii based on a moderate number of diving observations (but no laboratory trials) in South Africa. The latter two species occur in the eastern Atlantic and do not overlap in distribution with $L$. pealei. However, the adults are extremely similar in morphology, and hence the body patterns are one reasonable way to distinguish living animals. The western Atlantic $L$. plei and $L$. pealei have Lateral flame markings on the mantle, whereas the eastern Atlantic $L$. 
vulgaris, $L$. vulgaris reynaudii, and $L$. forbesi all have Lateral mantle streaks; the arrangement of chromatophores in the skin is very different and can be seen in preserved specimens. All five species seem to have highly comparable body patterns for crypsis and countershading, but differences appear in the intraspecific signals used during agonistic contests, courtship, and mate guarding. Sexual signals must be specific, and these are, therefore, the components of body patterns that will continue to provide unique markers, which is critical in distinguishing sympatric species.

\section{Conclusions}

Loligo pealei has an unexpectedly rich repertoire of body patterning. Any of the 34 chromatic components can be expressed instantly and in various combinations with the 5 postural and 12 locomotor components to produce each squid's wide variety of behavior. This is a unique capacity of cephalopods because of the direct neural control of hundreds of thousands of chromatophore organs in the skin. It also reflects this group's sensory capabilities and well-developed central nervous system (Hanlon and Messenger, 1996). In L. pealei, the largest portion of these visual signals seem to be used for intraspecific communication. This is not unexpected in a species that schools for much of its brief life, but it calls into question just how social squids are. Our findings in this report can be explained partly in the context of the life history and ecology of this species off Cape Cod. Loligo pealei individuals live less than a year (Brodziak and Macy, 1996), and their inshore migration each spring is generally thought to be linked to spawning. Off of south Cape Cod (which is a prime squid fishing area and much warmer than Cape Cod Bay and other locations northward), the squids arrive around the first week of May. The inshore trawl and weir trap fishery targets these schooling squids, which often have egg mops when captured, indicating high levels of spawning. This reproductive activity can be studied by divers throughout May, but it becomes increasingly difficult to find spawning congregations of squids around the southern Cape and islands (Nantucket and Vineyard Sounds) during the summer and fall, although eggs are trawled episodically throughout this time.

Our diving operations were designed to study sexual selection processes, thus our ethogram is based mostly on squids that were mature and actively engaged in agonistic contests between males, courting, mating, mate guarding, and egg laying. In May, many females already have sperm stored in the seminal receptacle, and it is likely that some reproductive behavior occurs offshore, before the squids migrate inshore. Moreover, the squids apparently spend considerable time in reproduction while inshore during the spring and summer, and thus it is not surprising that most of the components listed in Table I are associated with reproductive behavior. Our many hours (more than 440) of observation over three field seasons make us confident that the ethogram is quite complete for these activities and times. Whether other forms of social behavior occur remains to be discovered. For example, behaviors of young squids and of adults not engaged in reproductive activities during other times of the year and in different habitats have yet to be studied. However, we predict that such observations will reveal only a few new body patterns.

We have included polarization components in the ethogram largely because recent discoveries have shown that $L$. pealei (and probably all cephalopods) uses its visual polarization sensitivity to detect prey (Shashar et al., 1998) and produces polarization components in its skin that could be used for intraspecific signaling (Shashar and Hanlon, 1997; this paper, Fig. 3). Experiments on the cuttlefish Sepia officinalis suggested that it could possibly use this distinctive visual capability as a "hidden channel" of intraspecific communication (Shashar et al., 1996).

One of our recurrent and peculiar observations while diving was that aggregations of squids actively engaged in reproductive behaviors were usually conspicuous (i.e., bright white) rather than cryptic, thus potentially making them more easily detected by visual predators, which abound in the nearshore waters (e.g., mackerel, striped bass, flatfish). By helping squids avoid predators, schooling, combined with diurnal activity, may offset the disadvantage of increased visibility.

We believe that use of our ethogram will contribute to future behavioral studies demonstrating that $L$. pealei, like other loliginids, is a species with complex sexual behavior (Hanlon et al., 1997; Hanlon and Messenger, 1996; Sauer et al., 1997) that must be understood by those charged with protecting the resource. This species apparently has a window of opportunity for laying eggs that is restricted in both time (mainly spring) and space (shallow nearshore waters). Many squid fisheries worldwide target spawning congregations, so the predation pressure on spawners is increased (Hanlon, 1998). State and federal fishery managers estimate that stocks of $L$. pealei are being maximally exploited by commercial fishing (NEFSC, 1995). Understanding the mating system of such short-lived species will help managers assess the true effects of fishery practices that not only capture a large number of animals but, by removing spawning individuals, may disrupt the reproductive behavior of individuals and affect the recruitment and demographic structure of populations. 


\section{Acknowledgments}

We thank Mark Simonitsch, Ernie Eldridge, and Paul Lucas, who allowed us to dive in and around their weir traps to film squid spawning activity. We also thank numerous personnel of the Marine Resources Center of the MBL and many summer students who helped collect and feed squids. This work is the result of research sponsored in part by NOAA National Sea Grant College Program Office, Department of Commerce, under Grant No. NA86RG0075, Woods Hole Oceanographic Institution Sea Grant no. 22850012. Saltonstall-Kennedy Grant NA76FD0111 and NSF Grant IBN 9722805 also partially supported this work. KLB was partially funded by the Marine Models in Biological Research Program (NSF Grant DBI-9605155). ERL was supported by. ONR grant NR 4221022-01 and NSF grant 9419566. Special thanks to Rosie Davis who produced the first draft of Fig. 1.

\section{Literature Cited}

Arnold, J. M. 1962. Mating behavior and social structure in Loligo pealii. Biol. Bull. 123: 53-57.

Arnold, J. M. 1990. Squid mating behavior. Pp. 65-75 in Squid as Experimental Animals, D. L. Gilbert, W. J. Adelman, and J. M. Arnold, eds. Plenum Press, New York.

Brodziak, J. K. T., and W. K. Macy, III. 1996. Growth of longfinned squid, Loligo pealei, in the northwest Atlantic. Fish. Bull. 94: 212-236.

Cohen, A. C. 1976. The systematics and distribution of Loligo (Cephalopoda, Myopsida) in the western North Atlantic, with descriptions of two new species. Malacologia 15: 299-367.

Cooper, K. M., R. T. Hanlon, and B. U. Budelmann. 1990. Physiological color change in squid iridophores. II. Ultrastructural mechanisms in Lolliguncula brevis. Cell Tissue Res. 259: 15-24.

Cronin, T. W., N. Shashar, and L. Wolff. 1994. Portable imaging polarimeters. IEEE Proc. 12th IAPR Int. Conf. Pattern Recognition 606-609.

DiMarco, R. P., and R. T. Hanlon. 1997. Agonistic behavior in the squid Loligo plei (Loliginidae, Teuthoidea): fighting tactics and the effects of size and resource value. Ethology 103: 89-108.

Drew, G. A. 1911. Sexual activities of the squid, Loligo pealii (Les.). $J$. Morphol., Wistar Inst. of Anat. and Biol. 22: 327-359.

Driver, P. M., and D. A. Humphries. 1988. Protean Behaviour: The Biology of Unpredictability. Oxford University Press, New York.

Dubas, F., R. T. Hanlon, G. P. Ferguson, and H. M. Pinsker. 1986. Localization and stimulation of chromatophore motoneurons in the brain of the squid, Lolliguncula brevis. J. Exp. Biol. 121: 1-25.

Flamarique, I. N., and C. A. Hawryshyn. 1997. Is the use of underwater polarized light by fish restricted to crepuscular time periods? Vision Res. 37: 975-989.

Gilbert, D. L., W. J. Adelman, and J. M. Arnold, eds. 1990. Squid as Experimental Animals. Plenum Press, New York.

Griswold, C. A., and J. Prezioso. 1981. In situ observations on reproductive behavior of the long-finned squid, Loligo pealei. Fish. Bull. 78: 945-947.
Hanlon, R. T. 1982. The functional organization of chromatophores and iridescent cells in the body patterning of Loligo plei (Cephalopoda: Myopsida). Malacologia 23: 89-119.

Hanlon, R. T. 1988. Behavioral and body patterning characters useful in taxonomy of field identification of cephalopods. Malacologia 29: 247264.

Hanlon, R. T. 1996. Evolutionary games that squids play: fighting, courting, sneaking, and mating behaviors used for sexual selection in Loligo pealei. Biol. Bull. 191: 309-310.

Hanlon, R. T. 1998. Mating systems and sexual selection in the squid Loligo: How might commercial fishing on spawning squids affect them? Calif. Coop. Oceanic Fish Invest. Rep. 39: 92-101.

Hanlon, R. T., and J. B. Messenger. 1996. Cephalopod Behaviour. Cambridge University Press, Cambridge.

Hanlon, R. T., R. F. Hixon, and W. H. Hulet. 1983. Survival, growth, and behavior of the loliginid squid, Loligo plei, Loligo pealei and Lolliguncula brevis (Mollusca: Cephalopoda) in closed sea water systems. Biol. Bull. 165: 637-685.

Hanlon, R. T., K. M. Cooper, B. U. Budelmann, and T. C. Pappas. 1990. Physiological color change in squid iridophores. I. Behavior, morphology and pharmacology in Lolliguncula brevis. Cell Tissue Res. 259: 3-14.

Hanlon, R. T., M. J. Smale, and W. H. H. Sauer. 1994. An ethogram of body patterning behavior in the squid Loligo vulgaris reynaudii on spawning grounds in South Africa. Biol. Bull. 187: 1-10.

Hanlon, R. T., M. R. Maxwell, and N. Shashar, 1997. Behavioral dynamics that would lead to multiple paternity within egg capsules of the squid Loligo pealei. Biol. Bull. 193: 212-214.

Horvath, G., and D. Varju. 1997. Polarization pattern of freshwater habitats recorded by video polarimetry in red, green and blue spectral ranges and its relevance for water detection by aquatic insects. J. Exp. Biol. 200: 1155-1163.

Kattawar, G. W. 1994. Polarization of light in the ocean. Pp. 203-225 in Ocean Optics, R. W. Spinrad, K. L. Carder, and M. J. Perry, eds. Oxford University Press, New York.

McConathy, D. A., R. T. Hanlon, and R. F. Hixon. 1980. Chromatophore arrangements of hatchling loliginid squids (Cephalopoda, Myopsida). Malacologia 199: 279-288.

McKiernan, D. J., and D. E. Pierce. 1995. Loligo squid fishery in Nantucket and Vineyard Sounds. Mass. Div. Mar. Fish. Publ. No. 17648-75-200: 1-200.

NEFSC, Northeast Fisheries Science Center. 1995. Status of fishery resources off the northeastern United States for 1993. NOAA Tech. Mem. NMFS-FINEC. Woods Hole, Massachusetts. 1-138.

Packard, A., and F. G. Hochberg. 1977. Skin patterning in Octopus and other genera. Symp. Zool. Soc. Lond. 38: 191-231.

Porteiro, F. M., H. R. Martins, and R. T. Hanlon. 1990. Some observations on the behaviour of adult squids, Loligo forbesi, in captivity. $J$. Mar. Biol. Assoc. UK 70: 459-472.

Saidel, W. M., J. Y. Lettvin, and E. F. MacNichol. 1983. Processing of polarized light by squid photoreceptors. Nature 304: 534-536.

Sauer, W. H. H., M. J. Roberts, M. R. Lipinski, M. J. Smale, R. T. Hanlon, D. M. Webber, and R. K. O'Dor. 1997. Choreography of the squid's "nuptial dance." Biol. Bull. 192: 203-207.

Shashar, N., and R. T. Hanlon. 1997. Squids (Loligo pealei and Euprymna scolopes) can exhibit polarized light patterns produced by their skin. Biol. Bull. 193: 207-208.

Shashar, N., P. S. Rutledge, and T. W. Cronin. 1996. Polarization vision in cuttlefish--a concealed communication channel? J. Exp. Biol. 199: 2077-2084 
Shashar, N., R. T. Hanlon, and A. M. Petz. 1998. Polarization vision helps detect transparent prey. Nature 393: 222-223.

Stevenson, J. A. 1934. On the behavior of the long-finned squid (Loligo pealii, (Lesueur)). Can. Field-Nat. 48: 4-7.

Summers, W. C. 1983. Loligo pealei. Pp. 115-142 in Cephalopod Life Cycles, Vol. I, P. R. Boyle, ed. Academic Press, London.

Vecchione, M., T. F. Brakoniecki, Y. Natsukari, and R. T. Hanlon. 1998. A provisional generic classification of the family Loliginidae. Pp. 215-222 in Systematics and Biogeography of Ceph- alopods, Vol. I, N. Voss, M. Vecchione, R. B. Toll, and M. J. Sweeney, eds. Smithsonian Institution Press, Washington, DC.

Verrill, A. E. 1880-1881. The cephalopods of the northeastern coast of America. Part II. The smaller cephalopods, including the 'squids' and the octopi, with other allied forms. Trans. Conn. Acad. Sci. 5: 259 446.

Wolff, L. B., and A. G. Andreou. 1995. Polarization camera sensors. Image Vis. Comput. 16: 497-510. 